\title{
BMJ Open Life-course socioeconomic status and breast and cervical cancer screening: analysis of the WHO's Study on Global Ageing and Adult Health (SAGE)
}

\author{
Tomi Akinyemiju, ${ }^{1,2}$ Kemi Ogunsina, ${ }^{1}$ Swati Sakhuja, ${ }^{1}$ Valentine Ogbhodo, ${ }^{1}$ \\ Dejana Braithwaite ${ }^{3}$
}

To cite: Akinyemiju T, Ogunsina K, Sakhuja S, et al. Life-course socioeconomic status and breast and cervical cancer screening: analysis of the WHO's Study on Global Ageing and Adult Health (SAGE). BMJ Open 2016;6: e012753. doi:10.1136/ bmjopen-2016-012753

- Prepublication history for this paper is available online. To view these files please visit the journal online (http://dx.doi.org/10.1136/ bmjopen-2016-012753)

Received 24 May 2016 Revised 6 October 2016 Accepted 13 October 2016

CrossMark

${ }^{1}$ Department of Epidemiology, University of Alabama at Birmingham, Birmingham, Alabama, USA ${ }^{2}$ Comprehensive Cancer Center, University of Alabama at Birmingham, Birmingham, Alabama, USA

${ }^{3}$ Division of Cancer Epidemiology, Department of Epidemiology and Biostatistics, University of California San Francisco, San Francisco, California, USA

Correspondence to Tomi Akinyemiju; tomiakin@uab.edu

\section{ABSTRACT}

Objectives: Socioeconomic differences in screening have been well documented in upper-income countries however, few studies have examined socioeconomic status (SES) over the life-course in relation to cancer screening in lower-income and middle-income countries. Here, we examine individual, parental and life-course SES differences in breast and cervical cancer screening among women in India, China, Mexico, Russia and South Africa.

Setting: Data from the WHO's Study on Global Ageing and Adult Health (SAGE) 2007-2008 data were used for survey-weighted multivariable regression analysis. We examined the association between individual, parental and life-course SES in relation to breast and cervical cancer screening using education-based and employment-based measures of SES.

Participants: 22283 women aged 18-65 years, recruited from China, India, Mexico, Russia and South Africa.

Results: Having a college degree (OR 4.18; $95 \% \mathrm{Cl}$ 2.36 to 7.40 ) increased the odds of breast cancer screening compared with no formal education. Women with higher parental SES were almost 10 times more likely to receive breast cancer screening (OR 9.84; $95 \% \mathrm{Cl} 1.75$ to 55.5 ) compared with women with low parental SES. Stable higher lifecourse (OR 3.07; $95 \% \mathrm{Cl} 1.96$ to 4.79) increased breast cancer screening by threefold and increased cervical cancer screening by more than fourfold ( $\mathrm{OR}$ 4.35; $95 \% \mathrm{Cl} 2.94$ to 6.45 ); however, declining lifecourse SES was associated with reduced breast cancer screening (OR 0.26; $95 \% \mathrm{Cl} 0.08$ to 0.79 ) compared to low life-course SES.

Conclusions: Higher individual, parental and lifecourse SES was positively associated with breast and cervical cancer screening, although education-based SES measures were stronger predictors of screening compared with employment-based measures. Improving knowledge of the benefits of cancer screening and integrating cancer screening into routine healthcare practice for low SES women are actionable strategies that may significantly improve screening rates in low-income and middle-income countries.

\section{Strengths and limitations of this study}

- The strengths of this study include the use of data from the multicountry, nationally representative and standardised WHO's Study on Global Ageing and Adult Health (SAGE) study. SAGE was designed to elicit response on a wide range of health-related questions and had very high response rates, which permitted robust assessment of comprehensive measures of life-course SES and cancer screening in multiple countries.

- One potential limitation of this study includes the use of self-reported screening data; however, since SAGE is a standardised survey of multiple health items, any recall bias of self-reported screening is unlikely to be differential with respect to country or SES.

- Another potential limitation involves SES and potential country-level differences in the ability of education-based or employment-based measures to capture the full range of SES. We used measures of education and employment to better capture variability in SES, as these are less vulnerable to recall bias or social desirability bias, and have been used in past studies as robust measures of SES.

\section{INTRODUCTION}

Breast and cervical cancers remain the most common malignancies in women worldwide. ${ }^{1}{ }^{2}$ In low-income and middle-income countries (LMICs), incidence and mortality due to these cancers have increased significantly in recent decades; for instance, the estimated mortality from breast cancer in LMICs increased from $\sim 150000$ in 1990 to over 325000 in 2012 and is projected to increase to over 500000 by the year 2030, whereas cervical cancer mortality is estimated to increase from $\sim 230000$ in 2012 to $\sim 363000$ deaths by $2030 .^{2}{ }^{3}$ Whereas in upper-income countries (UICs), the estimated mortality from breast cancer is 
projected to increase from 197000 in 2012 to over 243000 , whereas cervical cancer deaths are projected to increase from $\sim 36000$ in 2012 to over 41000 deaths by 2030, further widening the gap in cancer mortality between UICs and LMICs. ${ }^{3-7}$

According to the International Agency for Research on Cancer (IARC), $84 \%$ and $53 \%$ of new cervical and breast cancer cases, respectively, as well as $88 \%$ and $58 \%$ of cervical and breast cancer deaths, respectively, occur in LMICs. $^{2}{ }^{7-10}$ Concurrently, breast and cervical cancer mortality rates have declined significantly in UICs in the past few decades, a trend that has been attributed to widespread use of routine cancer screening and improved cancer treatment. ${ }^{1}{ }^{6}{ }^{11}$ Routine screening and timely diagnostic follow-up is key to early diagnosis of cancer at stages where treatment is cheaper, less toxic and more effective. ${ }^{12}$ IARC recommends mammograms for women aged 50-74 years and Pap smears for women from 25 years triennially, ${ }^{13}{ }^{14}$ however, low financial resources may account for non-compliance with screening guidelines. Other causes of non-compliance include poor implementation of comprehensive cancer control plans (in countries that have them), poor healthcare infrastructure to implement cancer screening programmes, low levels of health literacy regarding the importance of routine cancer screening and high prevalence of competing health issues. ${ }^{15}$ Prior studies in the USA have reported that socioeconomic status (SES) at the individual and parental levels, as well as over the entire lifecourse, strongly influences health outcomes ${ }^{16-20}$ and is associated with screening. ${ }^{21-24}$ The life-course approach to understanding cancer screening recognises the complex interplay of early life factors, including parental and individual SES in shaping health behaviour, either directly through financial resources and healthcare access or indirectly through awareness of cancer screening recommendations. ${ }^{25}$

Although some studies in LMICs have shown that low individual SES negatively influences cancer screening rates, ${ }^{26-29}$ none to the best of our knowledge have examined SES over the life-course in relation to breast cancer screening and cervical cancer screening. It remains unclear whether parental SES plays a role in adherence to cancer screening guidelines above and beyond individual SES, or whether this association depends on the measure of SES, that is, education or income measures, or based on maternal or paternal SES measures. The present analysis fills this gap by examining individual, parental and life-course SES in relation to breast and cervical cancer screening among adult women in India, China, Mexico, Russia and South Africa.

\section{METHODS}

Data source and sample population

Data for this cross-sectional analysis included women aged 18 years and older from the WHO's Study on Global Ageing and Adult Health (SAGE) conducted in
China, Mexico, India, South Africa, Ghana and Russia in 2007-2008. SAGE is a longitudinal study of adults from nationally representative samples in these countries and aimed to evaluate disease risk factors, access to healthcare, health status and well-being. The current analysis focuses on five countries: China, India, Mexico, Russia and South Africa, one from each continent, to allow examination of middle-income countries that have experienced major economic and health transitions over the past few decades and face rising burden of noncommunicable diseases.

\section{Cancer screening outcomes:}

Key self-reported outcomes were: (1) receipt of screening mammography in the past 5 years among women aged 40 years and older and (2) receipt of a pelvic examination and Pap smear in the past 3 years among women aged 21 years and older.

\section{Socioeconomic status}

Individual and parental SES (maternal and paternal) were assessed based on education and employment measures. Educational attainment was based on highest level of education completed, and categorised as no formal education, primary school only, high school graduate and college or higher degree. Employment status was based on if individual and/or parent (maternal and paternal) is employed-in the public sector, private sector, self-employed or informal employment-or unemployed for any reason-including homemakers, retirees and those unable to work.

\section{Life-course SES}

Change in SES, that is, social mobility, from parent to individual was assessed based on education and employment measures. Life-course SES was defined based on maternal and paternal SES in relation to individual SES separately. Education-based life-course SES was categorised based on whether the parent or daughter completed a primary school education into: stable low, $<$ primary parental education and < primary daughter's education; declining, $\geq$ primary and $<$ primary; increasing, <primary and $\geq$ primary and stable high, $\geq$ primary and $\geq$ primary. Employment-based life-course SES was categorised based on whether the parent or the daughter was employed or unemployed into: stable low, parent and the daughter were unemployed; declining, parent was employed and the daughter was unemployed; increasing, parent was unemployed but the daughter was employed and stable high, both were employed.

\section{Statistical analysis}

Descriptive analysis was conducted to assess the distribution of sociodemographic, SES and life-course SES variables among study participants. Breast and cervical cancer screening rates by SES and life-course SES were also assessed overall and in each country. Other study covariates examined included age, marital status, rural/ 
urban residence and current health status. To account for the SAGE sampling strategy, each country was assigned household and individual-level analysis weights, which adjusts for sample selection and poststratification factors, using the most recent estimates of each country's population. All statistical analyses included these weights to ensure the generalisability of study results to individuals in the selected countries. Survey-weighted multivariable logistic regression models were created to determine the relationship between each SES and lifecourse SES variable in relation to breast and cervical cancer screening. Regression models were adjusted for age, marital status, rural/urban residence, health status and country to obtain adjusted estimates of the odds of breast and cervical cancer screening. For all analyses, $p$ values $\leq 0.05$ were considered statistically significant. All statistical analyses were performed with SAS V.9.4 (SAS Institute, Cary, North Carolina, USA).

\section{RESULTS}

Characteristics of the study population:

Among a total of 22283 women residing in five countries, almost half of women had at least a secondary school education $(43 \%)$, a third of women had no formal education (34\%), ranging from $58 \%$ in India, $28 \%$ in Mexico, 25\% in China, 23\% in South Africa to $1.3 \%$ in Russia (table 1). Other than Russia, where most women had parents with at least a secondary school education and employed in the public sector, the majority of women in other countries had mothers (58-80\%) and fathers (53-71\%) with no formal education, and most were either unemployed or self-employed. While $44 \%$ of women aged $\geq 21$ years received a pelvic examination in the past 3 years, out of this number, $56 \%$ had also received a Pap smear; however, only $28 \%$ of women aged $\geq 40$ years had received a mammogram in the past 5 years. Mammography screening ranged from $11 \%$ in India to $44 \%$ in Russia, whereas pelvic examinations ranged from $15 \%$ in India to $87 \%$ in Russia.

\section{SES and cancer screening:}

Breast cancer screening increased with increasing education, ranging from $10 \%$ among those with no formal education to $56 \%$ among those with a college education (table 2). Similar trends were observed based on maternal education; however, there was no clear gradient based on paternal education. Women employed in the public sector $(60 \%)$ or with maternal $(53 \%)$ or paternal (46\%) employment in the public sector had the highest screening rates. Breast cancer screening was low $(<10 \%)$ regardless of education among women in India, and highest among women with maternal self-employment $(19 \%)$, while screening increased markedly with increasing education among women in China, Mexico and Russia. In South Africa, screening rates were low among women with no formal education $(8 \%)$ and those with college education $(8 \%)$, including those with maternal
$(6 \%)$ and paternal $(8 \%)$ college education. Similarly, cervical cancer screening increased with increasing education overall, ranging from 5\% among those with no formal education to $51 \%$ among those with a college education. Cervical cancer screening was highest in Russia $(83-85 \%)$ and lowest in India $(<1-2 \%)$ across education levels, with clear positive gradients for individual, maternal and paternal education. Cervical cancer screening was highest among those employed in the public sector $(68 \%)$ across countries, ranging from $1.9 \%$ in India to $94 \%$ in Russia, while screening was lowest among women who were self-employed (29\%), ranging from $2 \%$ in India to $60 \%$ in Mexico.

\section{Life-course SES and cancer screening}

Significant differences in breast and cervical cancer screening were observed based on life-course SES (table 3). Breast cancer screening was higher among women with either stable high $(52 \%)$ or increasing $(32 \%)$ life-course SES based on maternal education, and among those with stable high $(45 \%)$ or increasing (32\%) life-course SES based on paternal education (all $\mathrm{p}$ values $<0.001)$. Breast cancer screening was also higher among women with stable $(56 \%)$ or increasing (49\%) life-course SES based on maternal employment and among those with stable (55\%) or increasing (50\%) lifecourse SES based on paternal employment. However, breast cancer screening was lowest among women with declining life-course SES (5\%) based on mother's education. Cervical cancer screening was also higher among women with stable high $(55 \%)$ or increasing (23\%) lifecourse SES based on maternal education, and among women with stable high $(45 \%)$ or increasing $(24 \%)$ lifecourse SES based on paternal education. The highest cervical cancer screening rate was observed among women with stable high life-course SES based on maternal employment (74\%) and paternal employment $(68 \%)$, and the lowest screening rates are observed among women with declining life-course SES based on mother's education $(6 \%)$ and among women with stable low life-course SES based on paternal's education (5\%).

\section{Multivariable adjusted model of SES and cancer screening:}

After adjusting for age, health status, rural/urban residence and marital status, having a college degree or higher (OR 4.18; 95\% CI 2.36 to 7.40 ) or secondary school education (OR 1.86; 95\% CI 1.27 to 2.78 ) was associated with higher odds of breast cancer screening compared with those with no formal education (table 4). Having a parent with a secondary school education (mother OR 2.50; 95\% CI 1.60 to 3.92; father OR 2.48; $95 \%$ CI 1.73 to 3.55 ) or higher increased the odds of breast cancer screening. In addition, women who themselves (OR 2.38; 95\% CI 1.60 to 3.53), had mothers (OR 2.39; $95 \%$ CI 1.60 to 3.59 ) or fathers (OR $1.83 ; 95 \%$ CI 1.21 to 2.78 ) employed in the public sector were significantly more likely to receive screening. Similarly, having 
Table 1 Baseline characteristics of female SAGE participants*

\begin{tabular}{|c|c|c|c|c|c|c|}
\hline & $\begin{array}{l}\text { Total } \\
\mathrm{N}=22283\end{array}$ & $\begin{array}{l}\text { China } \\
\mathrm{N}=8002\end{array}$ & $\begin{array}{l}\text { Mexico } \\
N=1689\end{array}$ & $\begin{array}{l}\text { India } \\
\mathrm{N}=7489\end{array}$ & $\begin{array}{l}\text { South Africa } \\
N=2427\end{array}$ & $\begin{array}{l}\text { Russia } \\
\mathrm{N}=2676\end{array}$ \\
\hline \multicolumn{7}{|l|}{ Age group } \\
\hline$<21$ & $512(4.6)$ & $13(1.2)$ & $5(0.3)$ & 483 (9.5) & $9(1.9)$ & $2(0.4)$ \\
\hline $21-40$ & $3234(37.1)$ & 400 (29.9) & $159(51.2)$ & 2424 (45.3) & $124(42.0)$ & $127(31.2)$ \\
\hline $40-65$ & $11451(47.0)$ & 4730 (58.3) & 704 (38.0) & 3357 (36.6) & $1390(46.8)$ & $1270(46.2)$ \\
\hline$>65$ & 7086 (11.3) & 2859 (10.6) & $821(10.5)$ & 1225 (10.6) & 904 (9.2) & 1277 (22.2) \\
\hline \multicolumn{7}{|l|}{ Marital status } \\
\hline Married & 14621 (77.3) & $6315(89.4)$ & 869 (64.9) & 5375 (77.8) & $874(37.9)$ & $1188(53.6)$ \\
\hline Never married & $1341(7.6)$ & $101(3.7)$ & 186 (19.9) & 483 (8.3) & 453 (34.0) & $118(8.1)$ \\
\hline Widow/divorced & $6321(15.1)$ & 1586 (6.9) & $634(15.2)$ & 1631 (13.9) & $1100(28.1)$ & $1370(38.3)$ \\
\hline \multicolumn{7}{|l|}{ Highest education } \\
\hline No formal education & $10341(34.5)$ & $3911(24.5)$ & $879(27.8)$ & 4402 (57.9) & 1053 (22.8) & $96(1.3)$ \\
\hline Primary school & 3311 (14.1) & 1301 (16.2) & $356(30.8)$ & $954(16.0)$ & 492 (15.6) & $208(2.6)$ \\
\hline Secondary school & 6311 (42.9) & $2493(50.5)$ & 218 (31.0) & 1265 (22.0) & $473(52.1)$ & $1862(76.8)$ \\
\hline College/university & $1321(8.4)$ & $297(8.8)$ & 169 (10.4) & $260(4.1)$ & $86(9.4)$ & 509 (19.2) \\
\hline \multicolumn{7}{|l|}{ Employment status } \\
\hline Unemployed & $8686(24.1)$ & 3746 (27.9) & 368 (15.9) & $1382(14.9)$ & $1438(38.3)$ & $1752(37.0)$ \\
\hline Private sector & $962(6.9)$ & $268(9.3)$ & $70(7.8)$ & $273(3.5)$ & 221 (11.0) & $130(1.5)$ \\
\hline Public sector & 1438 (14.3) & 489 (16.0) & 41 (3.3) & $116(1.5)$ & 103 (11.3) & $689(49.4)$ \\
\hline Self-employed & $11197(54.7)$ & $3499(46.8)$ & $1210(73.0)$ & $5718(80.1)$ & $665(36.4)$ & $105(4.6)$ \\
\hline \multicolumn{7}{|l|}{ Health status } \\
\hline Good & 7152 (45.1) & 1636 (12.3) & $626(49.4)$ & 1076 (11.4) & 908 (54.8) & $340(36.0)$ \\
\hline Moderate & $11075(42.8)$ & 2614 (49.5) & $856(40.6)$ & $3749(45.7)$ & 1140 (33.0) & $1578(50.5)$ \\
\hline Bad & 4056 (12.1) & 3752 (38.2) & 207 (10.0) & $1076(11.4)$ & 379 (12.2) & 758 (13.5) \\
\hline \multicolumn{7}{|l|}{ Mother's education } \\
\hline No formal & 17341 (73.3) & $7040(80.0)$ & 1442 (81.2) & $6145(87.2)$ & $1719(58.0)$ & $995(19.1)$ \\
\hline Primary school & $1546(9.4)$ & 342 (10.7) & 107 (10.3) & $382(6.5)$ & 162 (22.9) & $553(10.3)$ \\
\hline Secondary school & 1809 (14.9) & $325(10.4)$ & $38(6.0)$ & $323(5.8)$ & $142(13.5)$ & $981(59.4)$ \\
\hline College/university & $289(2.4)$ & $42(0.9)$ & $35(2.5)$ & $31(0.6)$ & $43(5.6)$ & $138(11.2)$ \\
\hline \multicolumn{7}{|l|}{ Father's education } \\
\hline No formal & $14815(58.4)$ & 6095 (65.2) & 1407 (71.1) & $4886(67.0)$ & $1575(52.8)$ & $852(16.4)$ \\
\hline Primary school & 2437 (13.1) & 772 (14.7) & 138 (19.3) & 777 (12.5) & 217 (12.7) & 533 (10.3) \\
\hline Secondary school & 3106 (23.9) & 766 (17.2) & $48(7.5)$ & 1012 (17.3) & $190(27.8)$ & $1090(60.4)$ \\
\hline College/university & $592(4.6)$ & $130(2.80$ & $29(2.2)$ & 204 (3.2) & $42(6.6)$ & 187 (12.9) \\
\hline \multicolumn{7}{|l|}{ Mother's employment } \\
\hline Unemployed & 10064 (37.7) & $3382(30.6)$ & $1021(48.9)$ & $4194(54.2)$ & 1107 (38.9) & $360(6.8)$ \\
\hline Private sector & $993(3.4)$ & $162(2.3)$ & $72(5.8)$ & $266(3.3)$ & 466 (18.3) & 27 (1.9) \\
\hline Public sector & $3272(20.0)$ & 895 (17.5) & $38(3.9)$ & 67 (1.0) & $87(9.4)$ & 2185 (89.3) \\
\hline Self-employed & 79549 (38.9) & $3563(49.6)$ & $558(41.3)$ & $2962(41.5)$ & 767 (33.4) & $104(2.1)$ \\
\hline \multicolumn{7}{|l|}{ Father's employment } \\
\hline Unemployed & 3128 (8.9) & 2230 (19.3) & $357(12.7)$ & $134(1.3)$ & $314(9.9)$ & $93(2.0)$ \\
\hline Private sector & 2049 (7.2) & $261(4.30$ & 207 (20.9) & $640(7.9)$ & $897(41.2)$ & $44(2.5)$ \\
\hline Public sector & $5223(28.0)$ & $1761(27.8)$ & $133(8.1)$ & 705 (9.3) & $244(9.5)$ & $2380(92.0)$ \\
\hline Self-employed & $11883(55.9)$ & 3750 (48.6) & $992(58.3)$ & $6010(81.4)$ & $972(39.4)$ & 159 (3.5) \\
\hline \multicolumn{7}{|l|}{ Pelvic examination } \\
\hline$<3$ years & 7831 (44.1) & 3165 (61.4) & 1108 (70.1) & 878 (14.9) & 587 (34.7) & 2093 (86.7) \\
\hline$\geq 3$ years & 14452 (55.9) & 4837 (38.6) & 581 (29.9) & $6611(85.1)$ & 1840 (65.3) & 583 (13.3) \\
\hline \multicolumn{7}{|l|}{ Pap smeart } \\
\hline Yes & 5769 (55.8) & $1776(43.1)$ & 1134 (93.6) & $129(17.8)$ & $549(77.6)$ & 2181 (91.3) \\
\hline No & 3238 (43.1) & 2252 (56.9) & $64(6.4)$ & $503(81.5)$ & $176(3.6)$ & $243(6.1)$ \\
\hline \multicolumn{7}{|l|}{ Mammogram $\ddagger$} \\
\hline$<5$ years & 4908 (27.7) & $2138(38.4)$ & $716(32.1)$ & 660 (10.8) & 396 (15.6) & $998(44.1)$ \\
\hline$\geq 5$ years & $17375(73.5)$ & $5864(61.6)$ & 973 (67.9) & 6829 (89.2) & $2031(84.4)$ & 1678 (55.9) \\
\hline
\end{tabular}


Table 2 Breast and cervical cancer screening rates by country and SES

\begin{tabular}{|c|c|c|c|c|c|c|c|c|c|c|c|c|}
\hline \multirow[b]{2}{*}{ SES (\%) } & \multicolumn{6}{|c|}{ Breast cancer screening* } & \multicolumn{6}{|c|}{ Cervical cancer screening $\dagger$} \\
\hline & $\begin{array}{l}\text { China } \\
(n=4946)\end{array}$ & $\begin{array}{l}\text { India } \\
(n=3640)\end{array}$ & $\begin{array}{l}\text { Mexico } \\
(n=755)\end{array}$ & $\begin{array}{l}\text { South } \\
\text { Africa } \\
(n=1472)\end{array}$ & $\begin{array}{l}\text { Russia } \\
(n=1320)\end{array}$ & $\begin{array}{l}\text { Total } \\
(n=12 \text { 133) }\end{array}$ & $\begin{array}{l}\text { China } \\
(n=53466)\end{array}$ & $\begin{array}{l}\text { India } \\
(\mathrm{n}=6064)\end{array}$ & $\begin{array}{l}\text { Mexico } \\
(n=9144)\end{array}$ & $\begin{array}{l}\text { South } \\
\text { Africa } \\
(n=1596)\end{array}$ & $\begin{array}{l}\text { Russia } \\
(n=1447)\end{array}$ & $\begin{array}{l}\text { Total } \\
(n=15367)\end{array}$ \\
\hline \multicolumn{13}{|l|}{ Own education } \\
\hline No formal education & 27.4 & 0.8 & 52.4 & 7.6 & 6.4 & 10.2 & 16.4 & 0.8 & 67.4 & 10.6 & 83.2 & 5.1 \\
\hline Primary school & 31.5 & 0.7 & 56.7 & 19.4 & 14.1 & 20.2 & 20.5 & 1.8 & 76.1 & 28.4 & 70.8 & 12.5 \\
\hline Secondary/high School & 43.7 & 0.6 & 52.8 & 37.1 & 52.1 & 41.9 & 32.4 & 1.8 & 57.3 & 28.7 & 88.3 & 39.6 \\
\hline $\begin{array}{l}\text { College/university/ } \\
\text { postgraduate }\end{array}$ & 83.7 & 0.2 & 66.3 & 7.8 & 54.5 & 55.7 & 48.2 & 1.6 & 69.3 & 38.1 & 85.4 & 51.1 \\
\hline \multicolumn{13}{|l|}{ Mother's education } \\
\hline No formal education & 35.1 & 0.8 & 53.6 & 21.0 & 43.0 & 21.4 & 26.6 & 1.2 & 66.4 & 22.2 & 71.3 & 15.0 \\
\hline Primary school & 57.2 & 0.3 & 64.5 & 16.0 & 48.9 & 44.5 & 34.7 & 1.6 & 71.5 & 27.1 & 84.1 & 31.1 \\
\hline Secondary/high school & 69.6 & 1.5 & 81.4 & 54.1 & 53.8 & 53.5 & 38.6 & 1.3 & 57.1 & 35.4 & 92.7 & 64.5 \\
\hline $\begin{array}{l}\text { College/university/ } \\
\text { postgraduate }\end{array}$ & 48.6 & 0.0 & 63.8 & 6.3 & 65.3 & 55.7 & 63.8 & 0.0 & 89.5 & 28.9 & 78.3 & 67.5 \\
\hline \multicolumn{13}{|l|}{ Father's education } \\
\hline No formal education & 32.8 & 0.7 & 52.4 & 23.5 & 43.5 & 20.4 & 23.4 & 1.3 & 64.3 & 18.7 & 77.1 & 13.9 \\
\hline Primary school & 47.8 & 1.6 & 74.1 & 12.8 & 39.9 & 31.1 & 35.3 & 0.8 & 84.8 & 50.8 & 85.0 & 27.0 \\
\hline Secondary/high school & 61.5 & 0.7 & 62.2 & 37.2 & 56.0 & 48.2 & 41.4 & 1.1 & 33.8 & 23.1 & 90.7 & 47.6 \\
\hline $\begin{array}{l}\text { College/university/ } \\
\text { postgraduate }\end{array}$ & 49.8 & 0.0 & 63.2 & 7.6 & 54.7 & 39.3 & 34.8 & 2.1 & 100.0 & 37.6 & 82.7 & 47.4 \\
\hline \multicolumn{13}{|l|}{ Own employment } \\
\hline Public sector & 68.9 & 0.0 & 55.8 & 6.4 & 59.2 & 60.3 & 46.6 & 1.9 & 59.7 & 61.8 & 93.8 & 67.6 \\
\hline Private sector & 46.6 & 1.8 & 34.1 & 13.3 & 47.3 & 37.1 & 43.2 & 0.9 & 69.0 & 49.2 & 83.2 & 42.6 \\
\hline Self-employed & 27.6 & 9.4 & 56.7 & 27.9 & 44.3 & 18.6 & 17.3 & 1.7 & 60.3 & 12.5 & 44.0 & 7.6 \\
\hline Unemployed & 40.8 & 0.7 & 57.6 & 23.5 & 40.1 & 29.5 & 32.3 & 2.8 & 59.9 & 23.9 & 82.2 & 29.5 \\
\hline \multicolumn{13}{|l|}{ Mother's employment } \\
\hline Public sector & 56.5 & 0.0 & 45.7 & 6.1 & 52.3 & 52.6 & 40.6 & 1.4 & 76.4 & 51.4 & 88.6 & 67.4 \\
\hline Private sector & 53.9 & 3.8 & 86.9 & 15.5 & 48.2 & 24.7 & 57.6 & 3.1 & 63.5 & 26.3 & 96.0 & 32.5 \\
\hline Self-employed & 34.9 & 19.3 & 59.3 & 15.7 & 57.4 & 29.3 & 19.7 & 0.9 & 69.9 & 32.3 & 58.2 & 12.4 \\
\hline Unemployed & 34.8 & 0.7 & 50.9 & 33.1 & 53.0 & 16.1 & 33.9 & 1.1 & 63.1 & 21.1 & 74.2 & 13.6 \\
\hline \multicolumn{13}{|l|}{ Father's employment } \\
\hline Public sector & 50.0 & 0.7 & 62.4 & 41.6 & 51.6 & 46.2 & 38.0 & 0.7 & 67.1 & 40.6 & 88.0 & 53.3 \\
\hline Private sector & 43.2 & 2.2 & 68.3 & 17.8 & 40.5 & 17.9 & 55.9 & 3.6 & 82.1 & 27.0 & 81.6 & 26.1 \\
\hline Self-employed & 35.2 & 8.7 & 54.2 & 22.2 & 71.2 & 21.3 & 19.0 & 0.9 & 60.0 & 29.3 & 81.4 & 8.9 \\
\hline Unemployed & 31.9 & 1.2 & 46.0 & 12.6 & 58.8 & 30.2 & 33.1 & 0.0 & 69.5 & 22.0 & 87.5 & 31.4 \\
\hline
\end{tabular}


Table 3 Breast and cervical cancer screening rates by life-course SES

\begin{tabular}{|c|c|c|c|c|c|}
\hline $\begin{array}{l}\text { Life-course SES } \\
\text { N (\%) }\end{array}$ & & $\begin{array}{l}\text { Breast cancer } \\
\text { screening }^{*}\end{array}$ & p Valuet & $\begin{array}{l}\text { Cervical cancer } \\
\text { screening }\end{array}$ & p Valuet \\
\hline Mother's education & Own education & & & & \\
\hline Greater than primary & Greater than primary & 819 (51.7) & \multirow[t]{4}{*}{$<0.0001$} & $1274(54.5)$ & \multirow[t]{4}{*}{$<0.0001$} \\
\hline Less than primary & Greater than primary & $1206(31.8)$ & & 1341 (22.9) & \\
\hline Greater than primary & Less than primary & $11(4.8)$ & & $14(5.3)$ & \\
\hline Less than primary & Less than primary & $979(15.7)$ & & $643(5.5)$ & \\
\hline Father's education & Own education & & & & \\
\hline Greater than primary & Greater than primary & $1074(45.3)$ & \multirow[t]{4}{*}{$<0.0001$} & $1513(44.6)$ & \multirow[t]{4}{*}{$<0.0001$} \\
\hline Less than primary & Greater than primary & 951 (31.9) & & 1102 (23.5) & \\
\hline Greater than primary & Less than primary & $43(10.9)$ & & $57(10.5)$ & \\
\hline Less than primary & Less than primary & $947(16.1)$ & & $600(4.9)$ & \\
\hline Mother's employment & Own employment & & & & \\
\hline Employed & Employed & $467(56.2)$ & \multirow[t]{4}{*}{$<0.0001$} & 749 (73.4) & \multirow[t]{4}{*}{$<0.0001$} \\
\hline Employed & Unemployed & $551(40.1)$ & & 757 (43.3) & \\
\hline Unemployed & Employed & 267 (48.9) & & 321 (37.4) & \\
\hline Unemployed & Unemployed & 1730 (19.3) & & $1445(9.6)$ & \\
\hline Father's employment & Own employment & & & & \\
\hline Employed & Employed & $563(54.6)$ & \multirow[t]{4}{*}{$<0.0001$} & 854 (68.4) & \multirow[t]{4}{*}{$<0.0001$} \\
\hline Employed & Unemployed & 825 (29.6) & & 1044 (28.3) & \\
\hline Unemployed & Employed & $171(50.4)$ & & 216 (37.9) & \\
\hline Unemployed & Unemployed & $1456(19.6)$ & & $1158(8.7)$ & \\
\hline
\end{tabular}

a secondary school (OR 2.24; $95 \%$ CI 1.52 to 3.30 ) or a college education (OR 4.18; 95\% CI 2.44 to 7.15), or having a mother (OR 2.34; 95\% CI 1.60 to 3.42) or a father (OR 2.13; $95 \%$ CI 1.55 to 2.94 ) with a secondary education, significantly increased cervical cancer screening after adjustment for potential confounders. Being employed in the public sector (OR 1.92; 95\% CI 1.31 to 2.81 ), or having a mother (OR 1.48; 95\% CI 1.04 to 2.12) employed in the public sector, significantly increased the likelihood of cervical cancer screening.

\section{Multivariable adjusted model of life-course SES and cancer screening}

In adjusted models, women with stable higher life-course SES based on maternal education, that is, high maternal education and high own education (OR 2.53; 95\% CI 1.69 to 3.80 ), or increasing life-course SES based on maternal education, that is, low maternal education and high own education (OR 1.23; 95\% CI 0.90 to 1.66), had greater odds of breast cancer screening (table 5). However, those with declining life-course SES had significantly lower odds of breast cancer screening (OR 0.26; $95 \%$ CI 0.08 to 0.79 ) compared with those with stable low life-course SES. Likewise, stable higher life-course SES based on father's education was associated with increased likelihood of breast cancer screening (OR $2.01 ; 95 \%$ CI 1.43 to 2.82). Similar associations were observed for cervical cancer screening among women with stable high life-course SES based on maternal education (OR 2.47; 95\% CI 1.47 to 4.16 ) and paternal education (OR 2.74; 95\% CI 1.66 to 4.54) and for increasing life-course SES based on education. Stable higher life-course SES based on maternal (OR 3.07; $95 \%$ CI 1.96 to 4.79 ) and paternal employment (OR 2.62; $95 \%$ CI 1.77 to 3.89) increased breast cancer screening by twofold to threefold and increased cervical cancer screening by more than fourfold (OR mothers 4.35; 95\% CI 2.94 to 6.45 ; OR fathers 4.24 ; $95 \%$ CI 2.95 to 6.11). Women with high education and high parental (maternal and paternal) education were almost 10 times more likely to receive breast cancer screening compared with those with at least one parent with low education (OR 9.84; $95 \%$ CI 1.55 to 55.5), and women who were employed with both parents also employed had a threefold higher odds of being screened (OR 3.18; 95\% CI 1.18 to 8.62$)$. Similarly, women who were employed and had both parents who were also employed had a fourfold increased likelihood of receiving cervical cancer screening (OR 4.02; 95\% CI 1.98 to 8.16) compared to unemployed women with both parents also unemployed.

\section{DISCUSSION}

This study provides a comprehensive assessment of the association between individual, parental and life-course SES in relation to breast and cervical cancer screening among women in China, India, Mexico, South Africa and Russia. In 2008, only $27 \%$ of women aged $\geq 40$ years had received a mammogram in the past 5 years, and only $23 \%$ of women aged $\geq 21$ years had received a 
Table 4 Multivariable logistic regression analysis of breast and cervical cancer screening by SES, SAGE 2008

\begin{tabular}{|c|c|c|c|c|}
\hline SES status* & $\begin{array}{l}\text { Breast cancer screening } \\
\text { OR }(95 \% \mathrm{Cl}) \dagger\end{array}$ & p Value & $\begin{array}{l}\text { Cervical cancer } \\
\text { screening OR }(95 \% \mathrm{Cl}) \dagger\end{array}$ & p Value \\
\hline \multicolumn{5}{|l|}{ Own education } \\
\hline College/university/postgraduate & 4.18 (2.36 to 7.40$)$ & $<0.00$ & $4.18(2.44$ to 7.15$)$ & $<0.00$ \\
\hline Secondary/high school & $1.86(1.27$ to 2.78$)$ & 0.53 & 2.24 (1.52 to 3.30$)$ & 0.09 \\
\hline Primary school & $1.17(0.73$ to 1.89$)$ & 0.01 & $1.34(0.84$ to 2.14$)$ & 0.02 \\
\hline No formal education & Ref. & Ref. & Ref. & Ref. \\
\hline \multicolumn{5}{|l|}{ Mother's education } \\
\hline College/university/postgraduate & 2.81 (1.13 to 6.97$)$ & 0.27 & $1.77(0.65$ to 4.85$)$ & 0.73 \\
\hline Secondary/high school & 2.50 (1.60 to 3.92$)$ & 0.18 & 2.34 (1.60 to 3.42$)$ & 0.03 \\
\hline Primary school & 1.97 (1.26 to 3.09$)$ & 0.91 & 1.38 (0.92 to 2.07$)$ & 0.55 \\
\hline No formal education & Ref. & Ref. & Ref. & Ref. \\
\hline \multicolumn{5}{|l|}{ Father's education } \\
\hline College/university/postgraduate & $1.94(0.91$ to 4.14$)$ & 0.59 & $1.66(0.80$ to 3.43$)$ & 0.84 \\
\hline Secondary/high school & 2.48 (1.73 to 3.55$)$ & 0.01 & 2.13 (1.55 to 2.94$)$ & 0.034 \\
\hline Primary school & 1.59 (1.07 to 2.36$)$ & 0.79 & 1.71 (1.20 to 2.44$)$ & 0.56 \\
\hline No formal education & Ref. & Ref. & Ref. & Ref. \\
\hline \multicolumn{5}{|l|}{ Own employment } \\
\hline Public sector & 2.38 (1.60 to 3.53$)$ & $<0.00$ & $1.92(1.31$ to 2.81$)$ & $<0.00$ \\
\hline Private sector & $1.10(0.66$ to 1.84$)$ & 0.86 & 1.36 (0.88 to 2.09$)$ & 0.03 \\
\hline Self-employed & 0.64 (0.48 to 0.86$)$ & $<0.00$ & $0.38(0.28$ to 0.53$)$ & $<0.00$ \\
\hline Unemployed & Ref. & Ref. & Ref. & Ref. \\
\hline \multicolumn{5}{|l|}{ Mother's employment } \\
\hline Public sector & 2.39 (1.60 to 3.59$)$ & 0.00 & 1.48 (1.04 to 2.12$)$ & 0.04 \\
\hline Private sector & $1.64(0.86$ to 3.14$)$ & 0.81 & 1.99 (1.13 to 3.50$)$ & 0.01 \\
\hline Self-employed & 1.47 (1.11 to 1.96$)$ & 0.66 & $0.54(0.39$ to 0.75$)$ & $<0.00$ \\
\hline Unemployed & Ref. & Ref. & Ref. & Ref. \\
\hline \multicolumn{5}{|l|}{ Father's employment } \\
\hline Public sector & 1.83 (1.21 to 2.78$)$ & 0.01 & $1.21(0.85$ to 1.74$)$ & 0.09 \\
\hline Private sector & 1.26 (0.68 to 2.34$)$ & 0.83 & 1.58 (0.89 to 2.80$)$ & 0.02 \\
\hline Self-employed & 1.30 (0.88 to 1.93$)$ & 0.92 & 0.51 (0.36 to 0.74$)$ & $<0.00$ \\
\hline Unemployed & Ref. & Ref. & Ref. & Ref. \\
\hline
\end{tabular}

pelvic examination with Pap smear in the past 3 years. There were clear SES gradients in screening rates and between-country differences in the association of SES and screening. For instance, although Russia had the highest proportion of women with a secondary school or college education (95\%), only $44 \%$ had received age-appropriate breast cancer screening. In contrast, only $41 \%$ of Mexican women had a secondary school or college education, yet over $30 \%$ of Mexican women had received age-appropriate breast cancer screening. Women with high SES defined based on education or public sector employment were most likely to have received breast or cervical cancer screening, and those with stable high or increasing life-course SES were more likely to be screened. Having a college degree or higher was by far the strongest individual-level predictor of screening, increasing the likelihood of breast or cervical screening by more than fourfold. In contrast, women who were self-employed or unemployed or who had maternal or paternal self-employment or unemployment were significantly less likely to receive screening. Stable high or increasing life-course SES was associated with increased cancer screening; however, declining lifecourse SES based on maternal education was associated with even lower odds of breast cancer screening compared with stable low life-course SES. Women who were educated and had both parents also educated had an almost 10-fold increase in the odds of breast cancer screening.

A growing number of studies have documented positive associations between childhood (or parental) and adult SES on health outcomes, ${ }^{15}$ 30-34 with low SES consistently liked with an increased risk of heart disease, diabetes, cancer and stroke. Low SES and the associated financial hardship may influence health outcomes through: (1) limited resources needed for disease prevention or health promotion activities, (2) the lack of knowledge about the health impact of lifestyle risk factors, behaviours or routine screening, (3) reduced access to healthcare due to financial, physical or social barriers to accessing the healthcare system and (4) psychosocial stress due to continued financial hardship. Cancer screening is likely influenced by consistently low SES via the lack of timely information about 
Table 5 Multivariable logistic regression analysis of breast and cervical cancer screening by life-course SES, SAGE 2008

\begin{tabular}{|c|c|c|c|c|c|}
\hline Life-course SES* & & $\begin{array}{l}\text { Breast cancer } \\
\text { screening OR } \\
(95 \% \mathrm{Cl}) \dagger\end{array}$ & p Value & $\begin{array}{l}\text { Cervical cancer } \\
\text { screening OR } \\
(95 \% \mathrm{Cl}) \dagger\end{array}$ & p Value \\
\hline Mother's education & Own education & & & & \\
\hline Greater than primary & Greater than primary & 2.53 (1.69 to 3.80$)$ & $<0.00$ & $2.47(1.47$ to 4.16$)$ & 0.08 \\
\hline Less than primary & Greater than primary & 1.23 (0.90 to 1.66$)$ & 0.12 & 1.67 (1.10 to 2.52$)$ & 0.99 \\
\hline Greater than primary & Less than primary & 0.26 (0.08 to 0.79$)$ & 0.00 & $1.89(0.43$ to 8.24$)$ & 0.82 \\
\hline Less than primary & Less than primary & Ref. & & Ref. & \\
\hline Father's education & Own education & & & & \\
\hline Greater than primary & Greater than primary & 2.01 (1.43 to 2.82$)$ & $<0.00$ & 2.7 (1.66 to 4.54$)$ & 0.01 \\
\hline Less than primary & Greater than primary & 1.08 (0.78 to 1.49$)$ & 0.96 & 1.72 (1.08 to 2.72 ) & 0.59 \\
\hline Greater than primary & Less than primary & $0.64(0.27$ to 1.56$)$ & 0.1 & 2.45 (1.12 to 5.39$)$ & 0.29 \\
\hline Less than primary & Less than primary & Ref. & & Ref. & \\
\hline Mother's employment & Own employment & & & & \\
\hline Employed & Employed & 3.07 (1.96 to 4.79 ) & 0.00 & 4.35 (2.94 to 6.45$)$ & $<0.00$ \\
\hline Employed & Unemployed & 1.68 (1.12 to 2.52$)$ & 0.40 & 1.83 (1.21 to 2.75$)$ & 0.24 \\
\hline Unemployed & Employed & 2.53 (1.68 to 3.82 ) & 0.07 & 2.90 (2.00 to 4.24$)$ & 0.06 \\
\hline Unemployed & Unemployed & Ref. & & Ref. & \\
\hline Father's employment & Own employment & & & & \\
\hline Employed & Employed & 2.62 (1.77 to 3.89$)$ & 0.00 & 4.24 (2.95 to 6.11$)$ & $<0.00$ \\
\hline Employed & Unemployed & $1.27(0.92$ to 1.76$)$ & 0.01 & 1.90 (1.36 to 2.66$)$ & 0.15 \\
\hline Unemployed & Employed & 2.66 (1.63 to 4.34$)$ & 0.02 & 3.32 (2.12 to 5.21$)$ & 0.02 \\
\hline Unemployed & Unemployed & Ref. & & Ref. & \\
\hline Both parent's education & Own education & & & & \\
\hline Both greater than primary & Greater than primary & 9.84 (1.75 to 55.5$)$ & 0.001 & $0.63(0.12$ to 3.20$)$ & 0.62 \\
\hline Both greater than primary & Less than primary & $1.22(0.13$ to 11.8$)$ & 0.18 & $0.32(0.03$ to 3.85$)$ & 0.50 \\
\hline$\geq 1$ less than primary & Greater than primary & 4.98 (0.91 to 27.3$)$ & 0.05 & $0.40(0.08$ to 1.98$)$ & 0.36 \\
\hline$\geq 1$ less than primary & Own less than primary & Ref. & & Ref. & \\
\hline Both parent employment & Own employment & & & & \\
\hline Both parent's employed & Employed & 3.18 (1.18 to 8.62$)$ & 0.001 & $4.02(1.98$ to 8.16$)$ & 0.001 \\
\hline Both parent's employed & Unemployed & $2.00(0.75$ to 5.36$)$ & 0.45 & $1.76(0.84$ to 3.70$)$ & 0.87 \\
\hline$\geq 1$ parent unemployed & Own employment & $1.44(0.55$ to 3.72$)$ & 0.24 & $1.21(0.61$ to 2.40$)$ & 0.01 \\
\hline$\geq 1$ parent unemployed & Unemployed & Ref. & & Ref. & \\
\hline
\end{tabular}

*Each life-course SES variable analysed in separate models adjusted for study covariates.

†Adjusted for smoking, alcohol, physical activity, rural/urban residence, marital status, country, age and health status.

SAGE, Study on Global Ageing and Adult Health; SES, socioeconomic status.

recommended cancer screening guidelines, the lack of financial resources to afford routine screening and limited availability of cheaper or subsidised screening programmes. Our findings provide evidence that there are major barriers to screening for low SES women in all the included countries; however, higher SES women are more successful in overcoming these barriers. Our observation that women with high SES based on education have the highest cancer screening rates suggests that health information is likely a critical factor- and improving access to and understanding of the importance of routine screening is a strategy that may help increase cancer screening among lower SES women. Additionally, women with higher SES based on employment, in particular public sector employment, also experienced high screening rates-suggesting that stable employment that may include healthcare benefits may improve screening through increased financial resources and/or better access to employment-based healthcare.

We observed strong associations between life-course SES and cancer screening, adding to the growing body of literature on the importance of early life and adult factors in human health, and cancer prevention, in particular. The consistently low cancer screening rates in resource-poor settings, particularly in many LMICs, ${ }^{35} 36$ and higher cancer screening rates in higher SES groups have been consistently reported. ${ }^{37-40}$ However, few studies have examined life-course SES in relation to cancer screening, ${ }^{41}$ with results showing that childhood conditions result in reduced probability of breast cancer screening. A recent study reported a higher risk of cancer in men with a downward social trajectory over the life-course compared with those at high social trajectory over the life-course. ${ }^{42}$ Other studies have also shown that declining life-course SES trajectory was associated with increased cancer-related risk factors, including reproductive behaviours and obesity. ${ }^{18}{ }^{43}$ Consistently, low SES over the life-course likely leads to cumulative disadvantage due to mechanisms, including low health literacy, poor access to high-quality healthcare, competing health risks, psychosocial stress and lack of financial resources for health, leading to stronger associations of 
life-course SES with health outcomes compared with SES measures at any single point in time. Although no formal definition for SES or life-course SES exists, education-based SES measures have emerged as stronger predictors of screening compared with employmentbased measures. This suggests that factors related to literacy, awareness of the benefits of screening, low selfefficacy regarding cancer prevention and early detection options may be more important for health outcomes, including cancer screening, ${ }^{26}{ }^{29}$ compared with income and financial factors. ${ }^{44}$ This is supported by our observation, as well as other studies showing a stronger influence of maternal education on cancer screening and daughter's adult health, ${ }^{43} 45$ that may be due to the fact that women may be strongly socialised and view their mothers as role models compared with their fathers. If highly educated mothers are more likely to receive screening or are well informed about the importance of routine cancer screening, this may positively shape daughters' own health-related behaviour, including cancer screening.

The observation that although $44 \%$ of women received a pelvic examination in the past 3 years but only $55 \%$ of those also received a Pap smear suggests that even when access to healthcare barriers are eliminated or reduced, appropriate screening still may not occur. Educating healthcare professionals on cancer screening guidelines and integrating cancer screening within routine healthcare settings may go a long way in increasing cancer screening rates, particularly for cervical cancer. While current recent recommendations for cervical cancer screening involve HPV DNA testing at ages 35 and/or 40, and HPV vaccination may contribute to significantly reducing the global burden of cervical cancer, ${ }^{46}$ these approaches still require significant initial investments in the healthcare infrastructure and substantial out of pocket costs to patients, limiting the immediate uptake of HPV testing as a routine cancer screening strategy. Regardless of screening method, community outreach programmes to improve knowledge of the importance of screening and increase cultural acceptability among low SES women will be important in all LMICs, while integration of routine screening into routine medical care and improved training of health personnel about communicating the benefits of screening to patients will likely increase cancer screening rates. Simply providing cancer screening technology, such as mammography machines, is unlikely to be sufficient to ensure successful utilisation by women at risk of cancer. $^{47}$ Additionally, national policies regarding screening may eliminate some of the structural barriers to screening. For instance, national cancer prevention policies that include financial subsidies for screening, free screening programmes and/or employment-based routine screening programmes may mitigate some of the effects of low SES on screening. In Russia, $66 \%$ of women employed in the public sector received breast and cervical cancer screening, and over $90 \%$ of women with maternal and paternal employment in the public sector had been screened for both cancers. This is in contrast with India and Mexico where comparable screening rates were $2.6 \%$ and $3.3 \%$, respectively, for public sector employment and likely reflect the lack of national comprehensive cancer screening programmes and/or integration of cancer screening into routine clinical practice. These between-country differences warrant further study, specifically country-level differences in healthcare infrastructure, health insurance coverage and screening costs, availability of medical personnel and population knowledge about cancer screening.

The strengths of this study include the use of data from the multicountry, nationally representative and standardised SAGE study. SAGE was designed to elicit response on a wide range of health-related questions and had very high response rates, which permitted robust assessment of comprehensive measures of lifecourse SES and cancer screening in multiple countries. One potential limitation of this study includes the use of self-reported screening data; however, since SAGE is a standardised survey of multiple health items, any recall bias of self-reported screening is unlikely to be differential with respect to country or SES. Another potential limitation involves SES and potential country-level differences in the ability of education or employment measures to capture the full range of SES. We used both measures of education and employment to better capture variability in SES, as these are less vulnerable to recall bias or social desirability bias, and have been used in past studies as robust measures of SES.

In summary, we observed SES gradients in breast and cervical cancer screening among women in China, India, Mexico, South Africa and Russia, and a stronger influence of life-course SES on screening. Future studies are needed to better understand and implement public health strategies focused on improving cancer screening rates among women with low SES over the life-course. For instance, targeted outreach programmes to increase knowledge of the benefits of cancer screening, integration of cancer screening within routine healthcare settings, as well as national-based and/or employment-based policies designed to mitigate SES differences in screening. Women with declining or low SES over the life-course experience cumulative disadvantage are least equipped to overcome financial and structural barriers to screening, and are also least likely to afford the financial catastrophe that often accompanies a late-stage cancer diagnosis.

Contributors TA conceived of the research question and statistical analysis and manuscript preparation. KO, SS and VO contributed to the statistical analysis and initial draft of the manuscript. DB contributed to the interpretation of results and writing of the manuscript.

Funding This work was supported by UAB Sparkman Center for Global Health.

Competing interests None declared.

Provenance and peer review Not commissioned; externally peer reviewed.

Data sharing statement No additional data are available. 
Open Access This is an Open Access article distributed in accordance with the Creative Commons Attribution Non Commercial (CC BY-NC 4.0) license, which permits others to distribute, remix, adapt, build upon this work noncommercially, and license their derivative works on different terms, provided the original work is properly cited and the use is non-commercial. See: http:// creativecommons.org/licenses/by-nc/4.0/

\section{REFERENCES}

1. Pecorelli S, Favalli G, Zigliani L, et al. Cancer in women. Int $J$ Gynaecol Obstet 2003;82:369-79.

2. Ferlay J, Soerjomataram I, Ervik M, et al. GLOBOCAN 2012 v1.0, Cancer Incidence and Mortality Worldwide. IARC CancerBase No. 11. Lyon, France: International Agency for Research on Cancer, 2013. http://globocaniarcfr (accessed 19 May 2014).

3. Tsu VD, Jeronimo J, Anderson BO. Why the time is right to tackle breast and cervical cancer in low-resource settings. Bull World Health Organ 2013:91:683-90.

4. Stevens GA, Mathers CD, Beard JR. Global mortality trends and patterns in older women. Bull World Health Organ 2013:91:630-9.

5. Sankaranarayanan R, Ramadas K, Qiao YL. Managing the changing burden of cancer in Asia. BMC Med 2014;12:3.

6. Reeler A, Qiao Y, Dare L, et al. Women's cancers in developing countries: from research to an integrated health systems approach Asian Pac J Cancer Prev 2009;10:519-26.

7. DeSantis CE, Bray F, Ferlay J, et al. International variation in female breast cancer incidence and mortality rates. Cancer Epidemiol Biomarkers Prev 2015;24:1495-506.

8. WCRF. World Cancer Research Fund data on specific cancers. 2015. http://www.wcrf.org/int/cancer-facts-figures/data-specificcancers

9. GLOBOCAN. Cancer Incidence and Mortality Worldwide in 2012. 2012. https://www.ncbi.nlm.nih.gov/pubmed/25220842

10. Ferlay J, Shin HR, Bray F, et al. Estimates of worldwide burden of cancer in 2008: GLOBOCAN 2008. Int J Cancer 2010;127:2893-917.

11. MalTKM. LEPM. Breast and Cervical Cancer Screening: Investing in Health Care Systems. Harvard Public Health Review 2015;5

12. Sankaranarayanan R, Boffetta P. Research on cancer prevention, detection and management in low- and medium-income countries. Ann Oncol 2010;21:1935-43.

13. Lauby-Secretan B, Scoccianti C, Loomis D, et al. Breast-cancer screening-viewpoint of the IARC Working Group. N Engl J Med 2015;372:2353-8.

14. Carter SM, Williams J, Parker L. Screening for cervical, prostate, and breast cancer interpreting the evidence. Am J Prev Med 2015;4:274-85

15. Mvundura M, Tsu V. Estimating the costs of cervical cancer screening in high-burden sub-Saharan African countries. Int J Gynaecol Obstet 2014:126:151-5.

16. Senese LC, Almeida ND, Fath AK, et al. Associations between childhood socioeconomic position and adulthood obesity. Epidemiol Rev 2009;31:21-51.

17. Chaparro MP, Koupil I. The impact of parental educational trajectories on their adult offspring's overweight/obesity status: a study of three generations of Swedish men and women. Soc Sci Med 2014;120:199-207.

18. Ball K, Mishra GD. Whose socioeconomic status influences a woman's obesity risk: her mother's, her father's, or her own? Int $J$ Epidemiol 2006;35:131-8.

19. Khang YH. [Lifecourse approaches to socioeconomic health inequalities]. J Prev Med Public Health 2005;38:267-75.

20. Adler NE, Newman K. Socioeconomic disparities in health: pathways and policies. Health Aff 2002;21:60-76.

21. NCl. Health disparities defined. Rockville, MD: U.S. Department of Health and Human Services, National Institutes of Health, National Cancer Institute, 2015

22. Pruitt SL, Shim MJ, Mullen PD, et al. Association of area socioeconomic status and breast, cervical, and colorectal cancer screening: a systematic review. Cancer Epidemiol Biomarkers Prev 2009;18:2579-99.

23. Bigby J, Holmes MD. Disparities across the breast cancer continuum. Cancer Causes Control 2005;16:35-44.
24. Fukuda Y, Nakamura K, Takano T. Reduced likelihood of cancer screening among women in urban areas and with low socio-economic status: a multilevel analysis in Japan. Public Health 2005; 119:875-84.

25. Lynch J, Smith GD. A life course approach to chronic disease epidemiology. Annu Rev Public Health 2005;26:1-35.

26. Chidyaonga-Maseko F, Chirwa ML, Muula AS. Underutilization of cervical cancer prevention services in low and middle income countries: a review of contributing factors. Pan Afr Med J 2015;21:231.

27. Gadgil A, Sauvaget C, Roy N, et al. Breast cancer awareness among middle class urban women-a community-based study from Mumbai, India. Asian Pac J Cancer Prev 2015;16:6249-54.

28. Yerramilli P, Dugee O, Enkhtuya $\mathrm{P}$, et al. Exploring knowledge, attitudes, and practices related to breast and cervical cancers in Mongolia: a National population-based survey. Oncologist 2015;20:1266-73.

29. Compaore S, Ouedraogo CM, Koanda S, et al. Barriers to cervical cancer screening in Burkina Faso: needs for patient and professional education. J Cancer Educ 2016;31:760-6.

30. Krishna Rao S, Mejia GC, Roberts-Thomson K, et al. Estimating the effect of childhood socioeconomic disadvantage on oral cancer in India using marginal structural models. Epidemiology 2015;26:509-17.

31. Tucker-Seeley RD, Li Y, Sorensen G, et al. Lifecourse socioeconomic circumstances and multimorbidity among older adults. BMC Public Health 2011:11:313.

32. Maty SC, Lynch JW, Raghunathan TE, et al. Childhood socioeconomic position, gender, adult body mass index, and incidence of type 2 diabetes mellitus over 34 years in the Alameda county study. Am J Public Health 2008;98:1486-94.

33. Smith GD, Hart C, Blane D, et al. Adverse socioeconomic conditions in childhood and cause specific adult mortality: prospective observational study. BMJ 1998;316:1631-5

34. Kaplan GA, Salonen JT. Socioeconomic conditions in childhood and ischaemic heart disease during middle age. BMJ 1990;301:1121-3.

35. Gakidou E, Nordhagen S, Obermeyer Z. Coverage of cervical cancer screening in 57 countries: low average levels and large inequalities. PLOS Med 2008;5:e132.

36. Knaul FM, Nigenda G, Lozano R, et al. Breast cancer in Mexico: a pressing priority. Reprod Health Matters 2008;16:113-23.

37. Akinyemiju TF. Socio-economic and health access determinants of breast and cervical cancer screening in low-income countries: analysis of the World Health Survey. PLOS ONE 2012;7:e48834.

38. Barton MB, Moore S, Shtatland E, et al. The relation of household income to mammography utilization in a prepaid health care system. $J$ Gen Intern Med 2001;16:200-3.

39. Duport N, Ancelle-Park R. Do socio-demographic factors influence mammography use of French women? Analysis of a French cross-sectional survey. Eur J Cancer Prev 2006;15:219-24.

40. Donnelly TT, Al Khater AH, Al Kuwari MG, et al. Do socioeconomic factors influence breast cancer screening practices among Arab women in Qatar? BMJ Open 2015;5:e005596.

41. Missinne S, Neels K, Bracke P. Reconsidering inequalities in preventive health care: an application of cultural health capital theory and the life-course perspective to the take-up of mammography screening. Sociol Health IIIn 2014;36:1259-75.

42. Schmeisser N, Conway DI, McKinney PA, et al. Life course social mobility and risk of upper aerodigestive tract cancer in men. Eur J Epidemiol 2010;25:173-82.

43. Pudrovska T, Anikputa B. The role of early-life socioeconomic status in breast cancer incidence and mortality: unraveling life course mechanisms. J Aging Health 2012;24:323-44.

44. Torssander J. Adult children's socioeconomic positions and their parents' mortality: a comparison of education, occupational class, and income. Soc Sci Med 2014;122:148-56.

45. Hill DH, Axinn WG, Thornton A. Competing hazards with shared unmeasured risk factors. Sociol Methodol 1993;23:245-77.

46. Kamangar F, Dores GM, Anderson WF. Patterns of cancer incidence, mortality, and prevalence across five continents: defining priorities to reduce cancer disparities in different geographic regions of the world. J Clin Oncol 2006;24:2137-50.

47. Wellensiek N, Moodley M, Moodley J, et al. Knowledge of cervical cancer screening and use of cervical screening facilities among women from various socioeconomic backgrounds in Durban, Kwazulu Natal, South Africa. Int J Gynecol Cancer 2002;12:376-82. 